\title{
Search for an association between neutrinos and radio-selected blazars with ANTARES
}

\section{Julien Aublin ${ }^{a, *}$ and Alexander Plavin ${ }^{b}$ on behalf of the ANTARES Collaboration} (a complete list of authors can be found at the end of the proceedings)

${ }^{a}$ Université de Paris, Laboratoire Astroparticules et Cosmologie, Paris, France

${ }^{b}$ Lebedev Physical Institute, Moscow, Russia

E-mail: julien.aublin@apc.in2p3.fr, alexander@plav.in

Recently, evidence for an association between high energy neutrinos detected by IceCube and radio-selected blazars has been found by Plavin et al.(2020, 2021). This result was achieved using an all sky complete sample of 3411 blazars selected on their parsec-scale flux density at $8 \mathrm{GHz}$ higher than $150 \mathrm{mJy}$. We perform a positional correlation analysis using the same sample of radioselected blazars, with the latest point source sample of neutrinos extracted from the data collected by the ANTARES detector between January 29, 2007 and February 28, 2020. Preliminary results are presented and discussed.

\footnotetext{
${ }^{*}$ Presenter
} 


\section{Introduction}

Almost ten years after the discovery of a diffuse astrophysical neutrino flux in 2013 by the IceCube Collaboration [1], the question of the origin of this excess is still not resolved. However, the recent studies using IceCube data ([2], [3], [4]), support the idea that extra-galactic sources, and in particular blazars could produce a detectable part of the observed high-energy neutrino flux.

The blazars are of particular interest because of the relativistic Doppler boost enhancing the flux of electromagnetic radiation and potentially associated neutrinos, making these object detectable up to cosmological distances. Previous searches have been conducted using the gamma-ray selected blazars from Fermi catalogs, with IceCube data [5], [6], [7] and with ANTARES [8].

Recently, a correlation between radio-selected blazars and the IceCube very high energy tracklike events has been reported in [9]. Moreover, the association has been confirmed in a second study using the lower-energy neutrinos from the IceCube $7 \mathrm{yr}$ public point source sample [10]. In the present study, the possible correlation between these radio-selected blazars and the neutrinos from the latest 13yr ANTARES point source sample is investigated.

\section{The blazar catalog}

The emission of high-energy neutrinos is a signature of hadronic interactions between accelerated charged particles and ambient matter or radiation. This processes could take place either in the central regions of galaxies: accretion disk, base of the jet; or in the outer and extended parts: kiloparsec-scale jets, blobs, or lobes and hot spots. Considering the distance of these sources, the angular resolution of present and future neutrino telescopes is not sufficient to differentiate between those acceleration regions.

The very long baseline interferometric (VLBI) radio measurement is a unique way to distinguish the emission of the central part of a galaxy from the outer parts, by resolving the central region of AGNs on a parsec scale, up to cosmological distances. The vast majority of the blazars used in the present analysis have a VLBI radio emission that is dominated by the base of the observed jet, and thus allows to test the for a possible neutrino emission from the central regions of AGN.

The $8 \mathrm{GHz}$ VLBI observations have been compiled by authors of [9] to provide a flux-limited complete subsample of AGNs on the full sky. The quantity $S_{8 \mathrm{GHz}}$, the flux density integrated over VLBI images at $8 \mathrm{GHz}$, is used to selected objects by requiring $S_{8 \mathrm{GHz}}>0.15 \mathrm{Jy}$. The complete sample contains 3411 objects, with 3027 in the field of view of ANTARES and available in the following link: http: //astrogeo.org/rfc/ (version being used: 2020d).

\section{The ANTARES data set}

The ANTARES neutrino telescope is a water Cherenkov detector that operates since 2007 on the bottom of the Mediterranean Sea, 40-km off-shore Toulon (France). The detection method is based on the observation of the Cherenkov light emitted in water by the relativistic particles produced by interactions of neutrinos in the vicinity of the instrumented volume [11]. 
The data sample used in the present analysis consists of 10162 track-like events after reconstruction and selection, that have been detected by ANTARES between January 29, 2007 and February 28,2020 , cumulating a total livetime of 3845 days. These events are almost totally induced by charged current interactions of $v_{\mu}$, producing a high energy muon that can propagate on large distances in water.

The event selection has been optimized for the detection of point-like sources, assuming a simple power-law energy spectrum $\propto E^{-2}$. A detailed description of the selection procedure can be found in [12].

For the current analysis, an updated time calibration has been used, the reconstruction and selection algorithms have been re-applied to the full ANTARES data to get the most accurate estimation of neutrino candidates arrival direction. The events contained in the previous 11yr point source sample used in [8] are not all included in this new 13yr sample as their updated reconstructed parameters may not fulfill the selection criteria. The overlap between the two samples is 7287 events, out of the 8754 present in the previous one.

The energy estimator used in the present analysis relies on the measure of muon energy deposit in water [13]. The selected tracks have estimated neutrino energies ranging from $\sim 100 \mathrm{GeV}$ to $\sim 1 \mathrm{PeV}$. The angular resolution depends on the energy and the topology of each event, but in average it is better than $0.4^{\circ}$ above $10 \mathrm{TeV}$. Due to the detector condition evolving with time (loss of optical modules, ageing of the photo-multipliers), the median angular resolution shows a significant variation over the years. This behavior is taken into account for the likelihood analysis presented in the section 5, by using Monte Carlo simulations [14] to build the pdfs separately for two different time periods: before 2014 and after 2014 .

\section{Counting method}

Following the approach used in [9], a simple method based on counting neutrino-blazar pairs is used as a first analysis. The quantity that is computed is the number of neutrinos that are located at an angular distance $\Psi$ from a blazar less than $x \cdot \beta$, where $\beta$ is the angular uncertainty coming from the neutrino reconstruction, and $x$ is a free parameter that is varied in the intervall $[0 ; 2]$.

This $x$ parameter is introduced to take into account a possible systematic difference between the output of the reconstruction algorithm and the true (unknown) angular error radius. With the help of Monte-Carlo simulations, it is however possible to study the relation between the error estimate $\beta$ and the true $68 \%$ containment radius $\Psi_{68 \%}$. For values $\beta \lesssim 0.5$, and reconstructed energies above $\sim 10 \mathrm{TeV}$, the relation between $\beta$ and $\Psi_{68 \%}$ is close to what is expected from a two-dimensional Gaussian function $\Psi_{68 \%} \simeq 2 \cdot \beta$. For higher values of $\beta$ or at lower energies, the value of $\Psi_{68 \%}$ starts to deviate from Gaussian behavior, by being significantly higher. The ANTARES point spread function (PSF), that is used in the following likelihood analysis, also shows the same significant difference compared to a Gaussian function.

The use of the $x$ parameter is then a simple empirical way to take into account this complicated behavior while still using the information of the quality of reconstruction of ANTARES neutrinos on an event-by-event basis. As the value of this parameter is scanned, a trial factor correction is applied to get a correct p-value estimation. 

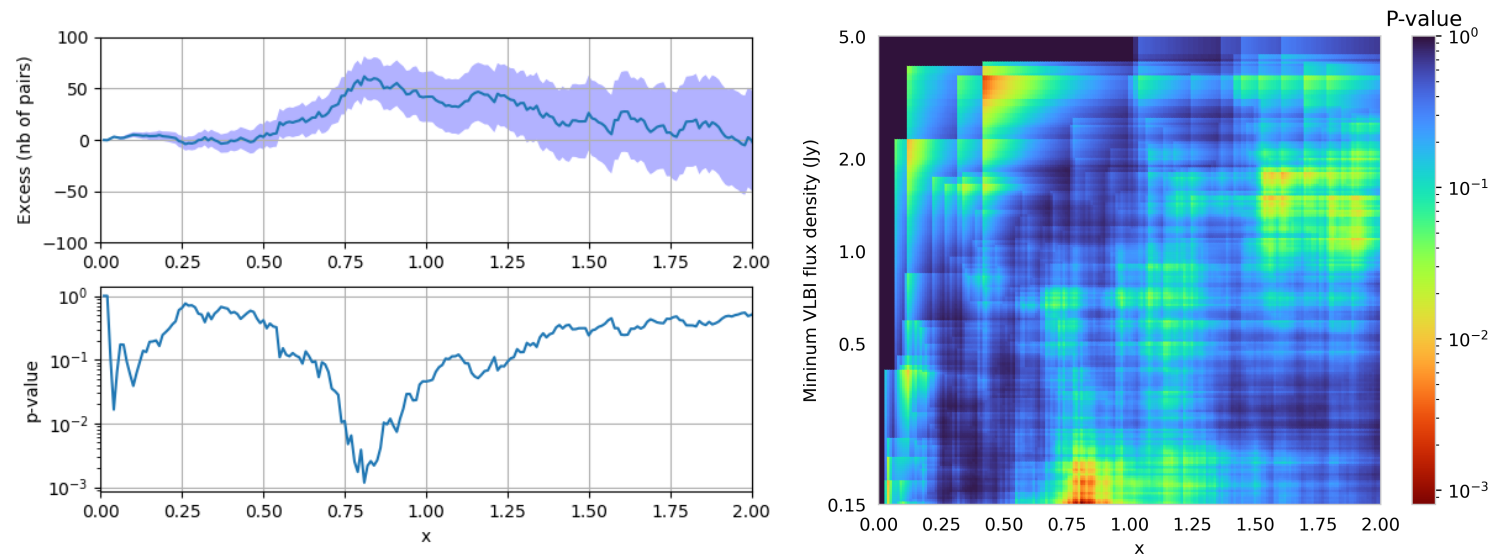

Figure 1: Left: result of the counting analysis with the full blazar catalog. The top panel shows the observed excess of pairs relative to random expectations, and the bottom panel shows the pre-trial p-value, as a function of the parameter $x$. Right: result of the scan over the radio flux density $S_{8 \mathrm{GHz}}$, the color code indicating the pre-trial p-value obtained for each value of the minimum flux density cut applied in the catalog and of the $x$ parameter defined in the text.

The result of the counting analysis applied to the full catalog of blazars and the total ANTARES $13 \mathrm{yr}$ point source sample is shown in figure 1 (left). The absolute minimum is found for $x=0.81$, where 451 pairs are observed in data, while 389 are expected from random simulations (62 pairs in excess). The associated pre-trial p-value is $p=1.210^{-3}(3.2 \sigma)$, leading after correction to a post-trial p-value of $P=2.210^{-2}(2.3 \sigma)$.

In [9], the blazars having associated IceCube neutrinos are found to have a higher than average radio flux density (time-integrated). The radio-neutrino correlation is therefore studied with ANTARES neutrinos by performing an additional scan on the radio flux density $S_{8 \mathrm{GHz}}$. Blazars are kept in the sample if they satisfy $S_{8 \mathrm{GHz}}>S_{\min }$.

The result of this two dimensional scan $\left(x, S_{\min }\right)$ is shown in figure 1 (right). The global minimum is found for $x=0.8$ and $S_{\min }=0.15 \mathrm{Jy}$, with a pre-trial p-value $p=1.210^{-3}$. This minimum corresponds to the previous findings of the one dimensional scan, and is obtained for the lowest value of the flux density cut, meaning that the whole blazar catalog is included. This means that the potential ANTARES neutrinos-blazar association is not eventually dominated by the high flux sources.

However, a second local minimum is observed for $x=0.45$ and $S_{\min }=3.7 \mathrm{Jy}$, with a pre-trial $\mathrm{p}$-value $p=3.210^{-3}$. This excess is caused by 4 neutrino-blazar pairs, while the expected number of chance coincidences is only 0.5 , with blazars brighter than $3.7 \mathrm{Jy}$ at separations below $0.45 \beta$. There are only 20 objets in the catalog that have a flux higher than $3.7 \mathrm{Jy}$. This motivates a manual inspection of the matching objects, their properties are listed in table 1.

All four neutrino candidate events can be considered notable: (i) two of them have high energies, top $0.3-0.6 \%$ of the whole dataset; (ii) two are associated with the same blazar J05384405; and (iii) the fourth event is associated with J1743-0350, one out of four likely associations with high-energy IceCube tracks found in [9].

The radio light curves measured in VLBI at 2 and $8 \mathrm{GHz}$ are shown in figure 2 for J05384405. There is a major flare visible around 2011-2012, and a sign of another flare in 2018 (end 


\begin{tabular}{cc|cccc}
\hline \multicolumn{2}{c|}{ Blazars } & \multicolumn{4}{c}{ Neutrinos } \\
\hline Source name & $S_{8 \mathrm{GHz}}(\mathrm{Jy})$ & Arrival time & Separation $\left[{ }^{\circ}\right]$ & $\beta$ & Estimated energy $(\mathrm{TeV})$ \\
\hline J0609-1542 & 3.76 & $2011-01-30$ & 0.15 & 0.47 & 70 \\
$\mathrm{~J} 1743-0350$ & 3.99 & $2019-03-08$ & 0.05 & 0.43 & 2.3 \\
& 4.2 & $2011-08-09$ & 0.39 & 0.93 & 45 \\
J0538-4405 & 4.2 & $2018-03-20$ & 0.34 & 0.82 & 6.0 \\
\hline
\end{tabular}

Table 1: Properties of the 4 neutrino-blazar pairs found for objects brighter than $3.7 \mathrm{Jy}$.

of observations). These periods are close to the dates of detected neutrinos, 2011-08-09 and 2018-03-20.

Additionally, J0538-4405 is a $\gamma$-bright source (labelled as 4FGL J0538.8-4405 in the Fermi 4FGL catalog [15]) and has a rich Fermi light-curve where strong $\gamma$-ray activity is apparent around 2010-2012. Unfortunately, the two ANTARES neutrino candidates have a large angular error $\beta$ value, that favors an atmospheric muon origin rather than an astrophysical origin.
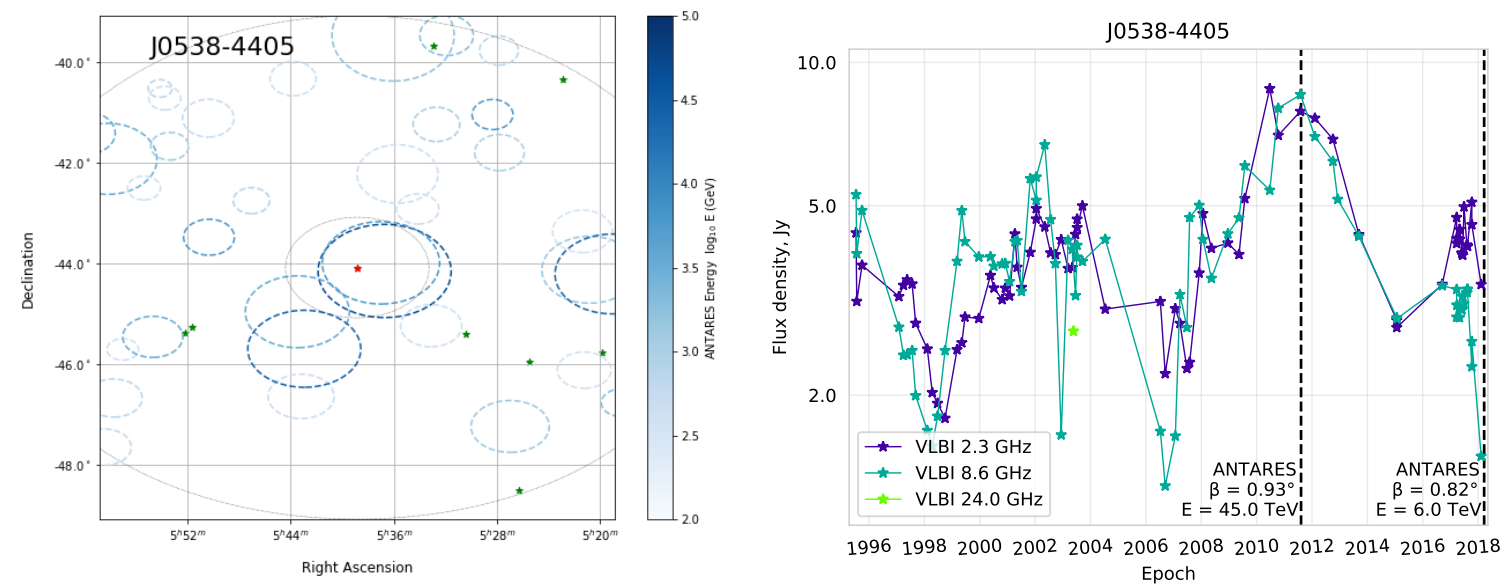

Figure 2: Left: arrival directions of ANTARES track events in equatorial coordinates around the position of the blazar J0538-4405. The neutrino events are represented by dashed empty circles with a radius equal to the angular error estimate $\beta$ while the estimated energy is indicated by the color code. The thin black circles centered around the source (red star marker) indicate the $1^{\circ}$ and $5^{\circ}$ angular distances to the source. The position of other blazars in the catalog are represented by a green star. Right: Radio light-curves of the blazar J0538-4405 measured in VLBI at three different frequency (see color code). The arrival times of the ANTARES neutrino candidates are indicated by a vertical dashed line.

The other two blazars J0609-1542 and J1743-0350, also experience radio flares close to the time of neutrino detection (see presentation). J1743-0350 flares both in 2019 when ANTARES received a neutrino, and in 2011 when the corresponding IceCube high-energy track occurred [9].

\section{Likelihood analysis}

A complementary likelihood analysis is presented here, making use of more information than the simple counting method about the neutrino candidates via the construction of energy and 
declination-dependent probability density functions. A pure power-law energy spectrum $E^{-\gamma}$ is assumed for the potential astrophysical neutrinos, and three values are tested $\gamma=2.0,2.25$ and 2.5.

The log-likelihood for both the null $H_{0}$ and the alternative $H_{1}$ hypotheses is written as:

$$
\begin{aligned}
& \ln \mathcal{L}\left(\mathrm{H}_{0}\right) \quad=\sum_{i}^{N} \ln \left(\mu_{b} B_{i}\right)-\mu_{b}, \\
& \ln \mathcal{L}\left(\mathrm{H}_{1}\right) \quad=\sum_{i}^{N} \ln \left(\mu_{s} S_{i}+\mu_{b} B_{i}\right)-\mu_{s}-\mu_{b},
\end{aligned}
$$

where $N$ is the total number of observed neutrino candidates, $S_{i}$ is the probability density function (PDF) of the signal and $B_{i}$ is the background PDF. The free parameters are the estimated number of signal $\mu_{s}$ and background $\mu_{b}$ events. The test statistic is then computed as: $\lambda=\ln \left(\frac{\max \left(\mathcal{L}\left(\mathrm{H}_{1}\right)\right)}{\max \left(\mathcal{L}\left(\mathrm{H}_{0}\right)\right)}\right)$.

The signal and background PDF are defined as the product of a spatial and an energy term:

$$
S_{i}=f_{s}\left(\alpha_{i}, \delta_{i}\right) \cdot g_{s}\left(E_{i}\right) \text { and } B_{i}=f_{b}\left(\delta_{i}\right) \cdot g_{b}\left(E_{i}\right),
$$

where $(\alpha, \delta)$ are the equatorial coordinates and $E$ the estimated energy of the neutrinos. The spatial term for the background is independent of the right ascension, and is estimated via a polynomial fit the real data $\sin \delta$ distribution. The signal term is obtained by summing over all the sources contributions:

$$
f_{s}\left(\alpha_{i}, \delta_{i}\right)=\frac{1}{\sum w_{j}} \sum_{j=1}^{N_{\text {sources }}} w_{j} \mathcal{F}_{j}\left(\alpha_{i}, \delta_{i}\right), \quad w_{j}=w_{j}^{\text {model }} \mathcal{A}\left(\delta_{j}\right),
$$

where $\mathcal{F}_{j}\left(\alpha_{i}, \delta_{i}\right)$ is the point spread function evaluated at the $j^{\text {th }}$ blazar's position, with weight $w_{j}$. The weight of the $j^{\text {th }}$ source takes into account the declination-dependent acceptance $\mathcal{A}(\delta)$ of the neutrino track sample computed for the corresponding $E^{-\gamma}$ energy spectrum. Two different weighting choices are considered: (i) an equal weight $w_{j}^{\text {model }}=1$, that could be considered as the default assumption, and (ii) a flux weight $w_{j}^{\text {model }}=S_{8 \mathrm{GHz}}$ to test the potential correlation between neutrino emission and radio flux density.

The point-spread function $\mathcal{F}_{E, \beta, \delta}$, defined as the probability density for the event direction to fall within a given angular distance from the true source direction, is obtained from Monte Carlo simulations, and has been parametrized for different energy, $\beta$ and declination intervals. This procedure is performed separately for events before and after 2014, to take into account the better detector performance in the early period.

The results of the likelihood analysis using the catalog of VLBI-selected blazars are summarized in table 2. For the full sample, the p-value obtained with the likelihood is between $5 \%$ and $10 \%$, depending on the assumed spectral index, the minimum being obtained for the softer energy spectrum and with a weight proportional to the radio flux density.

For comparision, those $\mathrm{p}$-values are a factor $\sim 2.5-5$ higher than the post-trial p-value found with the counting method, while for soft spectral indexes the number of fitted signal events $n_{s}$ is comparable to the 62 neutrino-blazar pairs in excess previously reported. When considering only blazars with a flux density $S_{8 \mathrm{GHz}}>3.7 \mathrm{Jy}$, the p-values found with the likekilhood are between $\sim(2-5) \times 10^{-3}$, in agreement with the p-value $p=3.210^{-3}$ found with the counting method. 
We note that differences in the resulting p-values between the two methods are to be expected, as the likelihood makes use of the additional energy information, and is based on Monte Carlo predictions for the point spread function.

\begin{tabular}{|c|c|c|c|c|c|c|c|}
\hline & & \multicolumn{3}{|c|}{ Equal weight } & \multicolumn{3}{c|}{ Flux weight } \\
\hline Sample & Spectral index & $n_{s}$ & $\lambda$ & p-value & $n_{s}$ & $\lambda$ & p-value \\
\hline \multirow{3}{*}{ Full VLBI } & $E^{-2}$ & 57 & 4.33 & 0.07 & 36 & 3.64 & 0.05 \\
& $E^{-2.25}$ & 112 & 7.26 & 0.08 & 64 & 5.14 & 0.06 \\
& $E^{-2.5}$ & 186 & 9.76 & 0.11 & 93 & 5.71 & 0.10 \\
\hline \multirow{3}{*}{$S_{8 \mathrm{GHz}}>3.7 \mathrm{Jy}$} & $E^{-2 .}$ & 8 & 4.84 & $2.310^{-3}$ & & & \\
& $E^{-2.25}$ & 10 & 5.16 & $2.510^{-3}$ & & & \\
& $E^{-2.5}$ & 11 & 4.84 & $4.510^{-3}$ & & & \\
& & & & & &
\end{tabular}

Table 2: Summary of the results obtained with the likelihood analysis: the fitted number of signal events $n_{s}$, the test statistic $\lambda$ and the p-value $p$ are reported for each combination of sample,spectral index and weighting choice.

\section{Discussion and conclusion}

A search for an association between VLBI radio-selected blazars and the arrival directions of the track-like events detected by ANTARES in 13 years of operation has been performed with two different methods, a simple counting technique and a more refined likelihood analyis. When searching on the full VLBI catalog, the two methods find an excess with p-values $p=0.02$ and $p \in[(2-5) \times 0.05-0.1]$ for the counting and the likelihood respectively.

An additional scan in radio-flux density shows that the bulk of the blazars contributing to this excess do not have a higher than average flux density (time-integrated). However, a second excess at very high flux is found with p-values $p=(2-5) \times 10^{-3}$, dominated by three blazars 0609-1542, J1743-0350 and J0538-4405, that are examined individually. The radio light-curves of these blazars show signs of flaring activity around the arrival time of neutrinos, especially for J0538-4405 that experiences a major augmentation of its radio flux over $\sim 4$ years.

An estimation of the p-value of the space and time coincidence between ANTARES neutrinos and radio emission has not yet been performed for those three sources, and will be addressed in a future study.

We advertise the special case of the blazar J0242+1101 that is presented in these conference [16], showing a hint of a spatial and temporal association between ANTARES neutrinos and a long radio flare, together with a gamma-ray flare observed by Fermi. The particular case will require a dedicated analysis to estimate the chance probability of the association between the VLBI, gamma-ray and neutrinos observations.

\section{References}

[1] ICECube Collaboration collaboration, Observation of high-energy astrophysical neutrinos in three years of IceCube data, Phys. Rev. Lett. 113 (2014) 101101. 
[2] P. Padovani, E. Resconi, P. Giommi, B. Arsioli and Y.L. Chang, Extreme blazars as counterparts of IceCube astrophysical neutrinos, Monthly Notices of the Royal Astronomical Society 457 (2016) 3582.

[3] A.F. S. Garrappa, S. Buson et al., Investigation of two Fermi-LAT gamma-ray blazars coincident with high-energy neutrinos detected by IceCube, Astrophys J. 880 (2019) 103.

[4] P. Giommi, T. Glauch, P. Padovani et al., Dissecting the regions around IceCube high-energy neutrinos: growing evidence for the blazar connection, Mon. Not. R. Astron. Soc. 497 (2020) 865.

[5] M.G. Aartsen, K. Abraham, M. Ackermann et al., The contribution of Fermi-2LAC blazars to difuse TeV-PeV neutrino flux, journal $=$ Astrophys J., year $=$ "2017", volume $=835$, eid $=45$, pages $=45$, doi $=10.3847 / 1538-4357 / 835 / 1 / 45$, .

[6] M. Huber and K. Krings, Results of IceCube searches for neutrinos from blazars using seven years of through-going muon data, PoS ICRC2017 Busan (2017) 994.

[7] M. Huber, Searches for steady neutrino emission from 3FHL blazars using eight years of IceCube data from the Northern hemisphere, PoS ICRC2019 Madison (2019) 916.

[8] A. Albert, M. André, M. Anghinolfi, G. Anton, M. Ardid, J.-J. Aubert et al., ANTARES search for point sources of neutrinos using astrophysical catalogs: A likelihood analysis, The Astrophysical Journal 911 (2021) 48.

[9] A. Plavin, Y.Y. Kovalev, Y.A. Kovalev and S. Troitsky, Observational evidence for the origin of high-energy neutrinos in parsec-scale nuclei of radio-bright active galaxies, The Astrophysical Journal 894 (2020) 101.

[10] A.V. Plavin, Y.Y. Kovalev, Y.A. Kovalev and S.V. Troitsky, Directional association of TeV to PeV astrophysical neutrinos with radio blazars, The Astrophysical Journal 908 (2021) 157.

[11] M. Ageron, J. Aguilar, I. Al Samarai, A. Albert, F. Ameli, M. André et al., ANTARES: The first undersea neutrino telescope, Nucl. Instrum. Methods. Phys. Res. A 656 (2011) 11-38.

[12] A. Albert, M. André, M. Anghinolfi et al., First all-flavor neutrino pointlike source search with the ANTARES neutrino telescope, Phys. Rev. D 96 (2017) 082001.

[13] J. Schnabel, Muon energy reconstruction in the ANTARES detector, Nucl. Instrum. Methods. Phys. Res. A 725 (2013) 106.

[14] A. Albert, M. André, M. Anghinolfi, G. Anton, M. Ardid, J.-J. Aubert et al., Monte carlo simulations for the ANTARES underwater neutrino telescope, Journal of Cosmology and Astroparticle Physics 2021 (2021) 064.

[15] M.A. S. Abdollahi, F. Acero et al., Fermi Large Area Telescope Fourth Source Catalog, Astrophys. J., Suppl. Ser. 247 (2020) 33.

[16] G. Illuminati, ANTARES search for neutrino flares from VLBI radio blazars, PoS ICRC 2021 Berlin (2021) . 


\section{Full Authors List: ANTARES Collaboration}

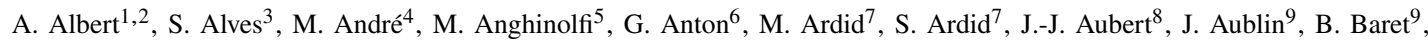

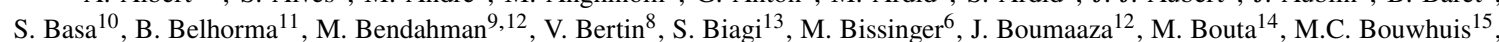
H. Brânzaş ${ }^{16}$, R. Bruijn ${ }^{15,17}$, J. Brunner ${ }^{8}$, J. Busto ${ }^{8}$, B. Caiffi ${ }^{5}$, A. Capone ${ }^{18,19}$, L. Caramete ${ }^{16}$, J. Carr ${ }^{8}$, V. Carretero ${ }^{3}$, S. Celli ${ }^{18,19}$, M. Chabab ${ }^{20}$, T. N. Chau ${ }^{9}$, R. Cherkaoui El Moursli ${ }^{12}$, T. Chiarusi ${ }^{21}$, M. Circella ${ }^{22}$, A. Coleiro ${ }^{9}$, M. Colomer-Molla ${ }^{9,3}$, R. Coniglione $^{13}$, P. Coyle ${ }^{8}$, A. Creusot ${ }^{9}$, A. F. Díaz ${ }^{23}$, G. de Wasseige ${ }^{9}$, A. Deschamps ${ }^{24}$, C. Distefano ${ }^{13}$, I. Di Palma ${ }^{18,19}$, A. Domi ${ }^{15,17}$, C. Donzaud ${ }^{9}, 25$, D. Dornic ${ }^{8}$, D. Drouhin ${ }^{1,2}$, T. Eberl ${ }^{6}$, T. van Eeden ${ }^{15}$, D. van Eijk ${ }^{15}$, N. El Khayati ${ }^{12}$, A. Enzenhöfer ${ }^{8}$, P. Fermani ${ }^{18,19}$, G. Ferrara $^{13}$, F. Filippini ${ }^{21,26}$, L.A. Fusco ${ }^{8}$, Y. Gatelet ${ }^{9}$, P. Gay ${ }^{27,9}$, H. Glotin $^{28}$, R. Gozzini ${ }^{3}$, R. Gracia Ruiz ${ }^{15}$, K. Graf ${ }^{6}$, C. Guidi ${ }^{5,29}$, S. Hallmann ${ }^{6}$, H. van Haren ${ }^{30}$, A.J. Heijboer ${ }^{15}$, Y. Hello ${ }^{24}$, J.J. Hernández-Rey ${ }^{3}$, J. Höß1 ${ }^{6}$, J. Hofestädt ${ }^{6}$, F. Huang ${ }^{8}$, G. Illuminati ${ }^{9}, 21,26$, C.W James ${ }^{31}$, B. Jisse-Jung ${ }^{15}$, M. de Jong ${ }^{15,32}$, P. de Jong ${ }^{15}$, M. Kadler ${ }^{33}$, O. Kalekin ${ }^{6}$, U. Katz ${ }^{6}$, N.R. Khan-Chowdhury ${ }^{3}$, A. Kouchner ${ }^{9}$, Y. Y. Kovalev $^{44,45,46}$, Yu. A. Kovalev ${ }^{44}$, I. Kreykenbohm ${ }^{34}$, V. Kulikovskiy ${ }^{5,36}$, R. Lahmann ${ }^{6}$, R. Le Breton ${ }^{9}$, D. Lefèvre ${ }^{35}$, E. Leonora ${ }^{36}$, G. Levi ${ }^{21,26}$, M. Lincetto ${ }^{8}$, D. Lopez-Coto ${ }^{37}$, S. Loucatos ${ }^{38,9}$, L. Maderer ${ }^{9}$, J. Manczak ${ }^{3}$, M. Marcelin ${ }^{10}$, A. Margiotta ${ }^{21,26}$, A. Marinelli ${ }^{39}$, J.A. Martínez-Mora ${ }^{7}$, K. Melis ${ }^{15,17}$, P. Migliozzi ${ }^{39}$, A. Moussa ${ }^{14}$, R. Muller ${ }^{15}$, L. Nauta ${ }^{15}$, S. Navas ${ }^{37}$, E. Nezri ${ }^{10}$, B. O'Fearraigh ${ }^{15}$, G.E. Păvălaş ${ }^{16}$, C. Pellegrino ${ }^{21,40,41}$, M. Perrin-Terrin ${ }^{8}$, V. Pestel ${ }^{15}$, P. Piattelli ${ }^{13}$, C. Pieterse ${ }^{3}$, A. V. Plavin ${ }^{44,45}$, A. Păun ${ }^{16}$, C. Poirè ${ }^{7}$, V. Popa ${ }^{16}$, T. Pradier ${ }^{1}$, N. Randazzo ${ }^{36}$, S. Reck ${ }^{6}$, G. Riccobene ${ }^{13}$, A. Romanov ${ }^{5,29}$, A. Sánchez-Losa ${ }^{3,22}$, F. Salesa Greus ${ }^{3}$, D. F. E. Samtleben ${ }^{15,32}$, M. Sanguineti ${ }^{5,29}$, P. Sapienza ${ }^{13}$, J. Schnabel ${ }^{6}$, J. Schumann ${ }^{6}$, F. Schüssler ${ }^{38}$, M. Spurio ${ }^{21,26}$, Th. Stolarczyk ${ }^{38}$, M. Taiuti ${ }^{5,29}$, Y. Tayalati ${ }^{12}$, S.J. Tingay ${ }^{31}$, S. V. Troitsky ${ }^{47}$, B. Vallage ${ }^{38,9}$, V. Van Elewyck ${ }^{9,41}$, F. Versari ${ }^{21,26,9}$, S. Viola ${ }^{13}$, D. Vivolo ${ }^{39,43}$, J. Wilms ${ }^{34}$, S. Zavatarelli ${ }^{5}$, A. Zegarelli ${ }^{18,19}$, J.D. Zornoza ${ }^{3}$, and J. Zúñiga ${ }^{3}$

${ }^{1}$ Université de Strasbourg, CNRS, IPHC UMR 7178, F-67000 Strasbourg, France. ${ }^{2}$ Université de Haute Alsace, F-68100 Mulhouse, France. ${ }^{3}$ IFIC - Instituto de Física Corpuscular (CSIC - Universitat de València) c/ Catedrático José Beltrán, 2 E-46980 Paterna, Valencia, Spain. ${ }^{4}$ Technical University of Catalonia, Laboratory of Applied Bioacoustics, Rambla Exposició, 08800 Vilanova i la Geltrú, Barcelona, Spain. ${ }^{5}$ INFN - Sezione di Genova, Via Dodecaneso 33, 16146 Genova, Italy. ${ }^{6}$ Friedrich-Alexander-Universität Erlangen-Nürnberg, Erlangen Centre for Astroparticle Physics, Erwin-Rommel-Str. 1, 91058 Erlangen, Germany. ${ }^{7}$ Institut d'Investigació per a la Gestió Integrada de les Zones Costaneres (IGIC) - Universitat Politècnica de València. C/ Paranimf 1, 46730 Gandia, Spain. ${ }^{8}$ Aix Marseille Univ, CNRS/IN2P3, CPPM, Marseille, France. ${ }^{9}$ Université de Paris, CNRS, Astroparticule et Cosmologie, F-75013 Paris, France. ${ }^{10}$ Aix Marseille Univ, CNRS, CNES, LAM, Marseille, France. ${ }^{11}$ National Center for Energy Sciences and Nuclear Techniques, B.P.1382, R. P.10001 Rabat, Morocco. ${ }^{12}$ University Mohammed V in Rabat, Faculty of Sciences, 4 av. Ibn Battouta, B.P. 1014, R.P. 10000 Rabat, Morocco. ${ }^{13}$ INFN - Laboratori Nazionali del Sud (LNS), Via S. Sofia 62, 95123 Catania, Italy. ${ }^{14}$ University Mohammed I, Laboratory of Physics of Matter and Radiations, B.P.717, Oujda 6000, Morocco. ${ }^{15}$ Nikhef, Science Park, Amsterdam, The Netherlands. ${ }^{16}$ Institute of Space Science, RO-077125 Bucharest, Măgurele, Romania. ${ }^{17}$ Universiteit van Amsterdam, Instituut voor Hoge-Energie Fysica, Science Park 105, 1098 XG Amsterdam, The Netherlands. ${ }^{18}$ INFN - Sezione di Roma, P.le Aldo Moro 2, 00185 Roma, Italy. ${ }^{19}$ Dipartimento di Fisica dell'Università La Sapienza, P.le Aldo Moro 2, 00185 Roma, Italy. ${ }^{20}$ LPHEA, Faculty of Science - Semlali, Cadi Ayyad University, P.O.B. 2390, Marrakech, Morocco. ${ }^{21}$ INFN - Sezione di Bologna, Viale Berti-Pichat 6/2, 40127 Bologna, Italy. ${ }^{22}$ INFN - Sezione di Bari, Via E. Orabona 4, 70126 Bari, Italy. ${ }^{23}$ Department of Computer Architecture and Technology/CITIC, University of Granada, 18071 Granada, Spain. ${ }^{24}$ Géoazur, UCA, CNRS, IRD, Observatoire de la Côte d'Azur, Sophia Antipolis, France. ${ }^{25}$ Université Paris-Sud, 91405 Orsay Cedex, France. ${ }^{26}$ Dipartimento di Fisica e Astronomia dell'Università, Viale Berti Pichat 6/2, 40127 Bologna, Italy. ${ }^{27}$ Laboratoire de Physique Corpusculaire, Clermont Université, Université Blaise Pascal, CNRS/IN2P3, BP 10448, F-63000 Clermont-Ferrand, France. ${ }^{28}$ LIS, UMR Université de Toulon, Aix Marseille Université, CNRS, 83041 Toulon, France. ${ }^{29}$ Dipartimento di Fisica dell'Università, Via Dodecaneso 33, 16146 Genova, Italy. ${ }^{30}$ Royal Netherlands Institute for Sea Research (NIOZ), Landsdiep 4, $1797 \mathrm{SZ}$ 't Horntje (Texel), the Netherlands. ${ }^{31}$ International Centre for Radio Astronomy Research - Curtin University, Bentley, WA 6102, Australia. ${ }^{32}$ Huygens-Kamerlingh Onnes Laboratorium, Universiteit Leiden, The Netherlands. ${ }^{33}$ Institut für Theoretische Physik und Astrophysik, Universität Würzburg, Emil-Fischer Str. 31, 97074 Würzburg, Germany. ${ }^{34}$ Dr. Remeis-Sternwarte and ECAP, Friedrich-Alexander-Universität Erlangen-Nürnberg, Sternwartstr. 7, 96049 Bamberg, Germany. ${ }^{35}$ Mediterranean Institute of Oceanography (MIO), Aix-Marseille University, 13288, Marseille, Cedex 9, France; Université du Sud Toulon-Var, CNRS-INSU/IRD UM 110, 83957, La Garde Cedex, France. ${ }^{36}$ INFN - Sezione di Catania, Via S. Sofia 64, 95123 Catania, Italy. ${ }^{37}$ Dpto. de Física Teórica y del Cosmos \& C.A.F.P.E., University of Granada, 18071 Granada, Spain. ${ }^{38}$ IRFU, CEA, Université Paris-Saclay, F-91191 Gif-sur-Yvette, France. ${ }^{39}$ INFN - Sezione di Napoli, Via Cintia 80126 Napoli, Italy. ${ }^{40}$ Museo Storico della Fisica e Centro Studi e Ricerche Enrico Fermi, Piazza del Viminale 1, 00184, Roma. ${ }^{41}$ INFN - CNAF, Viale C. Berti Pichat 6/2, 40127, Bologna. ${ }^{42}$ Institut Universitaire de France, 75005 Paris, France. ${ }^{43}$ Dipartimento di Fisica dell'Università Federico II di Napoli, Via Cintia 80126, Napoli, Italy. ${ }^{44}$ Lebedev Physical Institute of the Russian Academy of Sciences, Leninsky prospekt 53, 119991 Moscow, Russia ${ }^{45}$ Moscow Institute of Physics and Technology, Institutsky per. 9, Dolgoprudny, Moscow region, 141700, Russia. ${ }^{46}$ Max-Planck-Institut für Radioastronomie, Auf dem Hügel 69, 53121 Bonn, Germany. ${ }^{47}$ Institute for Nuclear Research, 60th October anniversary prospect 7a, Moscow 117312, Russia. 